\title{
131 THE EFFECT OF PENDER'S HEALTH PROMOTION MODEL IN IMPROVING THE NUTRITIONAL BEHAVIOR OF OVERWEIGHT AND OBESE WOMEN
}

Masoud Khodaveisi, ${ }_{1}^{1}$ Afsar Omidi, ${ }^{2}$ Shima Farokhi, ${ }^{3}$ Ali Reza Soltanian ${ }^{4} .{ }^{1}$ Chronic Diseases (Home Care) Research Center, and Community Health Nursing Department, Hamadan University of Medical Sciences, Hamadan, Iran; ${ }^{2}$ Community Health Nursing Department, Hamadan University of Medical Sciences, Hamadan, Iran; ${ }^{3}$ Chronic Diseases (Home Care) Research Center, and Community Health Nursing Department, Hamadan University of Medical Sciences, Hamadan, Iran; ${ }^{4}$ Modeling of Noncommunicable Disease Research Center, School of Public Heath, Hamadan University of Medical Sciences, Hamadan, Iran.

\subsection{6/bmjopen-2016-015415.131}

Background and aims: Changes in lifestyle and eating habits have put women at risk of obesity and overweight more than ever. This aim of this study was to investigate the effect of Pender's Health Promotion Model (HPM) to improve the nutritional behavior of overweight and obese women admitted to Fatemiyeh Hospital clinics in Hamadan, west Iran in 2015.

Methods: In this quasi-experimental study, 108 eligible women were selected and randomly assigned to two groups: one experimental and one control. Data were gathered using three questionnaires: demographics, Pender's HPM constructs, and nutritional behavior. The questionnaires were filled out by both groups as pre-test and two months later. A Pender's HPM-based intervention was conducted on the experimental group. The data were analyzed by paired and independent t-tests, ANCOVA, and Spearmans' correlation coefficient in SPSS/16. The level of significance was considered to be $<0.05$.

Results: The mean score of nutritional behavior was 41.75 \pm 3.28 and $42.36 \pm 3.69$ before the intervention and 79.09 \pm 5.27 and $49.72 \pm 9.49$ after the intervention in the experimental and control groups, respectively. The difference was significant only between before and after the intervention in the experimental group $(\mathrm{P}<0.001)$. Furthermore, the mean scores of the following variables were significantly different between before and after the intervention in the experimental group: nutritional behavior, perceived benefits, perceived self-efficacy, commitment to action, interpersonal and situational influences, affect related to behavior, and perceived barriers $(\mathrm{P}<0.001)$.

Conclusion: The results showed that Pender's HPM-based training improved nutritional behavior and some constructs of the model. Therefore, this educative model can be used by healthcare providers to improve the nutritional and other health promoting behaviors. 